\title{
What are the prognostic implications and factors relating to exercise induced electrocardiographic ST segment changes in the setting of a non-ischaemic stress echocardiogram?
}

\author{
Benjamin T. Fitzgerald ${ }^{1}$, Erin Smith ${ }^{1}$, and Gregory Scalia ${ }^{1}$ \\ ${ }^{1}$ GenesisCare Cardiology
}

January 4, 2022

\begin{abstract}
Background. Electrocardiographic (ECG) changes during stress testing are a common and perplexing finding during nonischaemic stress echocardiography (SE). Research has provided conflicting results regarding the implications. Methods. SE was performed after maximal Bruce protocol treadmill exercise. Results. 3020 consecutive patients, mean age $58 \pm 12$ years, $36 \%$ female, were followed-up for up to 9 years (mean $36 \pm 21$ months) post SE. Time to first cardiac event (composite of heart failure admission, worsening New York Heart Association class, worsening ejection fraction, acute coronary syndrome, revascularization, angina or cardiovascular death) was analyzed and adjusted using Cox proportional hazards regression. Prognostic significance was found with $1.5 \mathrm{~mm}$ of downsloping or horizontal ST depression. Adjusting for baseline differences, increased risk of composite major adverse cardiac events was shown with at least $1.5 \mathrm{~mm}$ of exercise induced ST depression (Hazard ratio [HR] of $2.47,95 \%$ Confidence ratio [CI $1.67-3.72, \mathrm{p}<0.0001$ ). Patients achieving high level exercise capacity ([?]13 metabolic equivalents or METs) with ST depression lower risk of cardiac events during follow-up Conclusion. Patients with ST segment depression but nonischaemic stress imaging have poorer prognosis compared to patients with non-ischaemic stress echocardiograms with normal stress ECGs. ST depression of $1.5 \mathrm{~mm}$ or more was established as a prognostically significance value. High exercise capacity improves prognosis, and ECG changes in that setting can be regarded as false positives. Overall, however, ST depression during non-ischaemic stress imaging is not a benign finding.
\end{abstract}

What are the prognostic implications and factors relating to exercise induced electrocardiographic ST segment changes in the setting of a non-ischaemic stress echocardiogram?

Dr Benjamin T. Fitzgerald, MBBS, a,b,c Erin Smith, BAppsSc(HMS), GradDip(Card Ultrasound), a,b and Professor Gregory M. Scalia, MBBS, MMedSc, FACC ${ }^{a, b, c, d}$

a. GenesisCare Cardiology, b. The Wesley Hospital, c. The Prince Charles Hospital, d. University of Queensland.

Address for correspondence:

Dr Ben Fitzgerald Ben.Fitzgerald@genesiscare.com

HeartCare Partners, GenesisCare, Level 5, Sandford Jackson Building, 30 Chasely Street, Auchenflower, Queensland, Australia, 4066. Telephone: + 6173648 2501, Fax: +61 732629631

Word Count: 2279

Abstract

Background. Electrocardiographic (ECG) changes during stress testing are a common and perplexing finding during non-ischaemic stress echocardiography (SE). Research has provided conflicting results regarding the 
implications.

Methods. SE was performed after maximal Bruce protocol treadmill exercise.

Results. 3020 consecutive patients, mean age $58 \pm 12$ years, $36 \%$ female, were followed-up for up to 9 years (mean $36 \pm 21$ months) post SE. Time to first cardiac event (composite of heart failure admission, worsening New York Heart Association class, worsening ejection fraction, acute coronary syndrome, revascularization, angina or cardiovascular death) was analyzed and adjusted using Cox proportional hazards regression. Prognostic significance was found with $1.5 \mathrm{~mm}$ of downsloping or horizontal ST depression. Adjusting for baseline differences, increased risk of composite major adverse cardiac events was shown with at least $1.5 \mathrm{~mm}$ of exercise induced ST depression (Hazard ratio [HR] of 2.47, 95\% Confidence ratio [CI] 1.67-3.72, $\mathrm{p}<0.0001$ ). Patients achieving high level exercise capacity ([?]13 metabolic equivalents or METs) with ST depression lower risk of cardiac events during follow-up

Conclusion. Patients with ST segment depression but non-ischaemic stress imaging have poorer prognosis compared to patients with non-ischaemic stress echocardiograms with normal stress ECGs. ST depression of $1.5 \mathrm{~mm}$ or more was established as a prognostically significance value. High exercise capacity improves prognosis, and ECG changes in that setting can be regarded as false positives. Overall, however, ST depression during non-ischaemic stress imaging is not a benign finding.

Key words: Stress echocardiography, ST depression, ischaemic heart disease, prognosis

Abbreviations:

CI - Confidence interval

ECG - electrocardiography

$\mathrm{EF}-$ Ejection fraction

FRS - Framingham risk score

HR - Hazard ratio

LAV - Left atrial volume

MACE - Major adverse cardiac events

METs - metabolic equivalents

NYHA - New York Heart Association

SE - stress echocardiography (SE)

This research did not receive any specific grant from funding agencies in the public, commercial, or not-forprofit sectors.

Introduction

Stress imaging was added to treadmill testing with the aim of increasing the accuracy of the investigative technique. [1-3] Subsequent research supported this proposal and initial papers suggested that the imaging result determined the prognostic outcomes rather than the electrocardiographic (ECG) findings. [2-5] Many clinicians were left with the suspicion that non-ischaemic stress imaging with significant ECG changes may not be completely reassuring. This research was conducted to test that hypothesis. Subsequently, one well conducted study has been published suggesting that abnormal exercise ECG findings in the setting of normal stress imaging may result in increased adverse events. [6]

Materials and Methods

Consecutive patients referred to GenesisCare Cardiology clinical testing facilities in Brisbane, Australia were studied prospectively between June 2011 to November 2015. Patients underwent standard Bruce 
protocol treadmill testing $[7,8]$ with digital gated echocardiography before and after exercise. Patients with an ischaemic stress echocardiogram, patients with a significant reduction in ejection fraction $(\mathrm{EF}<50 \%)$, those patients requiring dobutamine stress echocardiography, and patients with a paced rhythm or atrial fibrillation were excluded. Patients with a resting left bundle branch block were documented, but not included in the final analysis, due to difficulties in assessing ST change in that setting [9]. Indications for the test included chest pain and dyspnoea for investigation.

Exercise was replicated and quantitated using General Electric medical grade treadmills using Case systems (Milwaukee, USA). Standard Bruce protocols were used to produce exercise stress in a controlled and reproducible manner. Diagnostic ST segment change was defined as horizontal or downsloping ST depression 0.08 seconds from the J-point of at least $0.5 \mathrm{~mm}[7,8,10-12]$.

The echocardiographic image acquisition included the parasternal long axis, short axis, apical four, two, and three chambers. Ejection fraction was measured by Simpson's method. Stress imaging was completed in less than 90 seconds after cessation of exercise.

The echocardiography scanners utilised included the General Electric Vivid e9 (Horton, USA) and Vivid 7 (Horton, USA), Siemens SC2000 and SC2000 Prime (Mountain View, USA) and the Phillips ie33 and Epic (Best, The Netherlands). Metabolic equivalents (METs) were used to represent exercise capacity, as per standard protocols $[1,7,8,10]$.

The echocardiogram was performed by cardiac sonographers with subspecialty training in stress echocardiography. The stress ECG was monitored by an exercise physiologist. All tests were supervised and read by cardiologists with subspecialty training in stress echocardiography. Results were then over-read, standardized and recorded by a single stress echocardiography specialised cardiologist, blinded to the results and the outcomes.

Ischaemic stress echocardiograms were defined as those with new regional wall motion abnormalities in two or more contiguous segments, or cavity dilatation with a lack of cardiac augmentation (direct comparison of the pre- and post-exercise echocardiographic images). Non-ischaemic stress echocardiograms (niSE) were defined as having no evidence of myocardial ischaemia on the stress imaging (no new regional wall motion abnormalities, no cavity dilatation, and appropriate augmentation of cardiac contractility post exercise), with an appropriate augmentation of left ventricular function, as assessed by echocardiography.

In the follow-up phase, medical records were reviewed for up to nine (9) years after the stress echocardiogram had been performed. Predetermined cardiovascular end-points included admission to hospital with a diagnosis of heart failure, worsening New York Heart Association (NYHA) class, a reduction in ejection fraction of greater than $10 \%$, cardiovascular death, diagnosis of an acute coronary syndrome, new onset angina pectoris and percutaneous cardiac revascularisation. Worsening NYHA class, a diagnosis of acute coronary syndrome and angina were determined by the treating cardiologist at the time of follow-up. This assessment was made independent to the study, and the treating specialist was blinded to the results. The time interval to a patient's first significant cardiac event (a composite of the above) was analysed using Cox proportional hazards regression, adjusted for patient's age, pre-test Framingham risk (calculated and determined at the time of the stress echocardiogram), ejection fraction and left atrial volume.

The study design and methodology were reviewed and approved by the HeartCare Partners (GenesisCare) echocardiographic working group ethics subcommittee. The research protocol was carried out in accordance with The Code of Ethics of the World Medical Association (Declaration of Helsinki). All patients provided written consent.

Results

All non-ischaemic stress echocardiograms from June 2011 to November 2015 were prospectively analysed and followed. This resulted in a pool of 3020 studies. The patients were followed for up to 9 years or 48 354 patient months (mean of $36+-21$ months). The mean age was $58+-12$ years. There were 1087 females $(36 \%)$, which was expected for this cohort $[9,13,14]$. 
During follow-up, overall event rates were low. For all patients in this study, the event rate was $0.46 \%$ at 12 months, $1.0 \%$ at two years, $1.2 \%$ at three years and $2.8 \%$ at 5 years. This compared to a mean pre-test predicted Framingham risk score of $13.4+-10.3 \%$ over 10 years.

Patients who had any horizontal or downsloping ST depression ([?] $0.5 \mathrm{~mm}$ ) during niSE were shown to have more outcome events compared to patients without any ST depression (hazard ratio [HR] 1.55, 95\% confidence intervals $[95 \% \mathrm{CI}] 1.22-1.95, \mathrm{p}=0.0002)$. See Figure 1. However, comparison of the baseline characteristics of the two groups showed statistically significant differences, including age, medical history, Framingham risk score (FRS), ejection fraction (EF) and left atrial volume (LAV) (see Table 1). Adjusting for these baseline differences, a cut point of $1.5 \mathrm{~mm}$ of ST depression was shown to be incrementally prognostically significant for niSE (see Figure 2,3). These patients had statistically significantly more outcomes, compared to those with less or no ST depression. On the basis of these data, subsequent analyses were conducted using [?] $1.5 \mathrm{~mm}$ of exercise induced ST depression as the diagnostic value for an electrically positive stress test during niSE. An increased risk of major adverse cardiac outcomes (death, acute coronary syndromes and revascularisation) was shown for those with at least $1.5 \mathrm{~mm}$ of exercise induced ST depression with a HR of 2.47 (95\% CI 1.67-3.72, $\mathrm{p}<0.0001)$. See Figure 4.

Events were seen in $21.0 \%$ (95\% CI 0.17-0.25) of all niSE tests with $1.5 \mathrm{~mm}$ of ST depression. This compared to an event rate of $11.1 \%$ (95\% CI $0.10-0.12, \mathrm{p}<0.001)$ for patients who did not have ST depression, over the total follow up period. The majority events occurred later in the follow-up period.

Exercise capacity has previously been shown to effect outcomes post stress testing. [15,16]. Patients who were able to exercise to greater than or equal to 13 metabolic equivalents (METs, to the end of stage 4 on the standard Bruce protocol) during niSE, but with significant ST depression had a HR of 0.55 (95\% CI 0.39-0.77, $\mathrm{p}=0.005)$ compared to a HR of 1.83 (95\% CI 1.3-2.57) for those exercising to less than 13 METs with significant ST changes. See Figure 5. Exercise to more than 10.3 METs has previously been shown to have prognostic benefit [16]. In this study, those with diagnostic ST change ([?] 1.5mm) during niSE, exercise capacity of greater than 10.3 METs did not impart statistically different outcomes compared to those with lower exercise endurance. Reduced exercise capacity has been shown to result in poorer patient outcomes. [15] Unfortunately for patients with ST depression and lower exercise capacity (e.g. [?] 7.1 METs), there were insufficient numbers for an accurate analysis $(\mathrm{n}=77$ for patients with ST depression [?]1.5mm and [?] 7.1 METs).

\section{Discussion}

Stress echocardiography is an established and mature technique for the assessment of patients with suspected and established coronary artery disease. [1-3] Stress imaging significantly increases the accuracy of the test over exercise ECG analysis alone. [1-4] In the ischaemic cascade, regional wall abnormalities occur before ECG changes. [17] Non-ischaemic stress echocardiograms have been shown to have very low event rates in follow up [1-6] including the patients in this cohort (1.2\% for all patients at 3 years post testing). As a consequence, patients with ST changes with normal stress imaging have been felt to have a low risk result. [3,4] However, ST depression during exercise testing occurs frequently, in both men and women. [10] Concerns have remained over the significance of this finding.

The results here confirm the findings of Daubert et al [6] that ST changes during niSE result in more adverse outcomes despite reassuring stress imaging. A number of factors influence these findings, including age, pre-test risk, patient medical history and medical therapy. Differences persist despite correcting for these factors. Adverse events tend to occur later in follow-up. Additionally, the research presented here suggests that $1.5 \mathrm{~mm}$ of ST depression during niSE appears to be the prognostically significant amount of change. It also suggests that high exercise capacity has a moderating effect and that those patients have low risk going forward despite the abnormal ECG findings.

These results also confirm that non-ischaemic stress echocardiography overall confers a good prognostic result for patients. While there were increased events for patients with ST changes compared to those who did not, overall event rates were low. 
Why a non-ischaemic stress echocardiogram with ST segment change carries increased risk is more perplexing. According to the ischaemic cascade theory, ECG changes should be a later event than regional wall motion abnormalities. In treadmill stress echocardiography, imaging occurs after the patient has stopped exercising. There is a delay of 5-20 seconds before stress imaging can commence. It is possible that echocardiographic changes normalise in these patients before imaging occurs. Stress echocardiography accuracy is approximately 85\%. [1-3] It is possible that the non-ischaemic tests with ECG changes are false negatives, within the error rate of the test. Small vessel disease or ischaemia at a level smaller than the major epicardial vessels may be part of this story. [18,19] The study by Daubert et al, included patients with ischaemic stress echocardiograms. Those patients had significantly more events that patients with ECG changes but normal echocardiographic responses. [6] The adverse outcomes associated with stress ECG changes may be related to non-coronary causes (see below). It may be that ST segment change is another independent adverse prognostic marker during stress testing.

There are a number of causes for exercise related ST segment change during niSE that don't involve myocardial ischaemia. Repolarization and conduction abnormalities such as bundle branch block and pre-excitation can make interpretation of the stress ECG difficult [1,10, 20-22]. There were very few patients in this cohort with these changes, and they were not included in the analysis (pre-specified condition). Medications such as digoxin can have influence on the ECG [10,20-22]. No patients in this study were recorded to be on digoxin. Conditions such as hypertension, left ventricular hypertrophy, valvular heart disease and arrhythmia have been associated with increased risk of exercise induced ST changes [10, 20-22]. Age and an exaggerated blood pressure response are also noted to influence the stress ECG [10,20-22]. An attempt was made to account for these influences during analysis. In the basic assessment of stress testing, ECG changes are assumed to be related to coronary ischaemia.

There were limitations to this research. To definitively confirm the results of the stress echocardiogram, this research would need to be conducted with patients undergoing an anatomical test to confirm the diagnosis in a blinded manner after the stress test. From a practical and clinical viewpoint, invasive coronary angiography would negate the non-invasive attributes of stress echocardiography. There would also be ethical implications for intermediate risk patients undergoing an invasive test. [1,2 23] Computer tomography coronary angiography could be used, but would add significant cost and patient inconvenience. While complications are low, there is still risk. [23-24] Multiple previous studies have already confirmed the accuracy of stress imaging for the detection of myocardial ischaemia. [1-6] In most clinical scenarios, stress echocardiography is used to attempt to avoid additional testing. A limited number of patients in this study had a clinical reason to evaluate the coronary anatomy during follow-up, and in those patients, the effective and accurate role of stress echocardiography in this setting was confirmed.

In this non-randomized, single centre cohort study, there were documented differences at baseline between the groups (see Table 1). Attempts were made to take these differences into account, but these baseline differences may have influenced the outcomes presented here.

There was a low frequency of positive tests overall. There was a large attrition rate in patient follow-up after five (5) years. These factors will influence the results.

Evaluation of the medical records was a potential source of ascertainment bias. The possibility exists for events occurring and not being recorded in the patient records. While failure to appropriately account for missing data in analyses may lead to bias and loss of precision, imputation of missing results also requires assumptions. The present analysis of cases with complete data rather than imputation of missing values may not be associated in an epidemiological context with substantial bias in reported regression estimates. $[25]$

The multivariable prediction model described in the present study was derived from echocardiographic observations at a single centre. There were significantly less women than men (a common problem in cardiac research). This does reflect real world experiences. The reviewers were not blinded to the results of the stress test, making it possible for ascertainment or other biases to occur. Overall event rates were low. The 
present model would benefit from validation within an independently collected data set.

Conclusions

This study confirms that ST segment depression despite non-ischaemic stress echocardiographic imaging carries a poorer prognosis compared to patients with those with normal exercise ECG analysis. It suggests that $1.5 \mathrm{~mm}$ or more of ST depression during niSE has prognostic significance. High exercise capacity significantly improves prognosis, and suggests that ECG changes in that scenario can be regarded as false positives. The findings have clinical implications for physicians utilising these important investigative modalities. These data confirm, corroborate and expand upon the prognostic value of exercise stress echocardiography.

References:

1. Pellikka PA, Arruda-Olson A, Farooq A, et al. Guidelines for the Performance, Interpretation and Application of Stress Echocardiography in Ischemic Heart Disease: From the American Society of Echocardiography. J Am Soc Echocardiogr. 2020;33(1):1-41.

2. Marwick T.H. Stress Echocardiography - Its Role in the Diagnosis and Evaluation of Coronary Artery Disease. 2 ed. Boston: Kluwer Academic Publishers; 2003.

3. Marwick TH, Mehta R, Arheart K, Lauer MS. Use of exercise echocardiography for prognostic evaluation of patients with known or suspected coronary artery disease. J AmColl Cardiol . 1997;30(1):83-90.

4. Mahenthiran J, Bangalore S, Yao SS, Chaudhry FA. Comparison of prognostic value of stress echocardiography versus stress electrocardiography in patients with suspected coronary artery disease. Am J Cardiol . 2005;96(5):628-634.

5. Al-Mallah M, Alqaisi F, Arafeh A, Lakhdar R, Al-Tamsheh R, Ananthasubramaniam K. Long-term favourable prognostic value of negative treadmill echocardiogram in the setting of abnormal treadmill electrocardiogram: a 95 month median duration follow-up study. J Am Soc Echocardiogr. 2008;21(9):1018-1022.

6. Melissa A. Daubert, Joseph Sivak, Allison Dunning, Pamela S. Douglas, Brian Coyne, Tracy Y. Wang, Daniel B. Mark, Eric J. Velazquez. Implications of Abnormal Exercise Electrocardiography with Normal Stress Echocardiography. JAMA Intern Med. 2020 Apr; 180(4): 494-502.

7. Bruce RA, McDonough JR. Stress testing in screening for cardiovascular disease. Bull N Y Acad Med. 1969 Dec;45(12):1288-305.

8. Bruce RA, Kusumi F, Hosmer D. Maximal oxygen intake and nomographic assessment of functional aerobic impairment in cardiovascular disease. Am Heart J. 1973;84(4):546-562.

9. Gibbons RJ, Balady GJ, Beasley JW, Bricker JT, Duvernoy WF, Froelicher VF, Mark DB, Marwick TH, McCallister BD, Thompson PD Jr, Winters WL, Yanowitz FG, Ritchie JL, Gibbons RJ, Cheitlin MD, Eagle KA, Gardner TJ, Garson A Jr, Lewis RP, O'Rourke RA, Ryan TJ. ACC/AHA Guidelines for Exercise Testing. A report of the American College of Cardiology/American Heart Association Task Force on Practice Guidelines (Committee on Exercise Testing). J Am Coll Cardiol. 1997;30(1):260-311.

10. Fitzgerald BT, Scalia WM, Scalia GM. Female False Positive Exercise ECG Testing - Fact Versus Fiction. Heart, Lung and Circulation, 2019; 28:735-741

11. Ellestad MH, Savitz S, Bergdall D, Teske J. The False Positive Stress Test. American Journal of Cardiology. 1977;40:691-685.

12. Fortuin NJ, Weiss JL. Exercise Stress Testing. Circulation. 1977; 56(5):699-712.

13. Fitzgerald B.T, Presneill J.J, Scalia I.G, Hawkins C.L, Celermajer Y, Scalia W.M, Scalia G.M. The Prognostic Value of the Diastolic Stress Test in Patients Undergoing Treadmill Stress Echocardiography. J Am Soc Echocardiogr. 2019;32(10):1298-1306.

14. Fitzgerald BT, Logan JK, Weldon A, Kwon A, Scalia IG, Scalia GM. The prognostic value of estimating stroke volume before and after exercise treadmill stress echocardiography. Echocardiography. 2020; 37(11):1809-1819.

15. Morris CK, Ueshima K, Kawaguchi T, Hideg A, Froelicher VF. The prognostic value of exercise capacity: a review of the literature. Am Heart J. 1991;122(5):1423-31.

16. Snader CE, Marwick TH, Pashkow FJ, Harvey SA, Thomas JD, Lauer MS. Importance of Estimating 
Functional Capacity as a Predictor of All-Cause Mortality Among Patients Referred for Exercise Thallium Single-Photon Emission Computer Tomography: Report of 3400 Patients from a Single Center. J Am Coll Cardiol. 1997;30(3):641-8.

17. Nesto RW, Kowalchuk GJ. The ischemic cascade: temporal sequence of hemodynamic, electrocardiographic and symptomatic expressions of ischemia. Am J Cardiol. 1987;59(7):23C-30C.

18. Taqueti VR, Carli MF. Coronary Microvascular Disease Pathogenic Mchanisms and Therapeutic Options: JACC State-of-the-Art Review. JACC. 2018;72(21):2625-2641.

19. Berry C, Sidak N, Pereira AC, Ford TJ, Touyz RM, Kaski JC, Hainsworth AH. Small-Vessel Disease in the Heart and Brain: Current Knowledge, Unmet Therapeutic Need, and Future Directions. JAHA. 2019;8(3):1-11.

20. Detrano R, Salcedo E, Passalacqua M, Friis R. Exercise Electrocardiographic Variables: A Critical Appraisal. J Am Coll Cardiol. 1986; 8(4):836-47.

21. Hill J, Timmis A. Exercise tolerance testing. BMJ. 2002; 324(7345):1084-1087.

22. Carlen A, Gustafsson M, Astrom Aneq M, Nylander E. Exercise-induced ST depression in an asymptomatic population without coronary artery disease. Scandinavian Cardiovascular Journal. 2019;53(4):206-212

23. Braunwald's Heart Disease: A Textbook of Cardiovascular Medicine. 10 ed. 10 ed. Philadelphia: Saunders; 2015.

24. Lu MT, Douglas PS, Udelson JE, Adami E, Ghoshhajra BB, Picaard MH, Roberts R, Lee KL, et al. Safety of Coronary CT Angiography and Functional Testing for Stable Chest Pain in the PROMISE Trial: A Randomized Comparison of Test Complications, Incidental Findings and Radiation Dose. J Cardiovasc Comput Tomogr. 2017;11(5):373-382.

25. Hughes RA, Heron J, Sterne JAC, Tilling K. Accounting for missing data in statistical analyses: multiple imputation is not always the answer. Int J Epidemiol. 2019, Mar 16. doi: 10.1093/ije/dyz032

Tables.

Table 1. Baseline characteristics of patients with exertional ST depression compared to those with a normal stress ECG.

\begin{tabular}{llll}
\hline & ST depression with exertion & Normal stress ECG & $\mathrm{P}$ value \\
\hline Number & 497 & 2519 & \\
Age & $\mathbf{6 0 . 1} \pm \mathbf{1 1 . 2}$ years & $\mathbf{5 7 . 6} \pm \mathbf{1 2 . 7}$ & $\mathbf{p}<\mathbf{0 . 0 0 0 1}$ \\
Gender & $35 \%$ & $36 \%$ & $\mathrm{p}=0.35$ \\
BMI & $27.7 \pm 4.4 \mathrm{~kg} / \mathrm{m}^{2}$ & $28.1 \pm 5.5$ & $\mathrm{p}=0.07$ \\
BSA & $1.98 \pm 0.22 \mathrm{~m}^{2}$ & $1.99 \pm 0.24$ & $\mathrm{p}=0.42$ \\
Framingham risk score & $\mathbf{1 4 . 3} \pm \mathbf{1 0 . 8}$ & $\mathbf{1 3 . 0} \pm \mathbf{1 0 . 1}$ & $\mathbf{p}=\mathbf{0 . 0 1}$ \\
Hypertension & $52 \%$ & 42 & $\mathbf{p}=\mathbf{0 . 0 0 0 4}$ \\
LVH & 37 & 19 & $\mathbf{p}<\mathbf{0 . 0 0 0 1}$ \\
Coronary artery disease & 18 & 9 & $\mathbf{p}<\mathbf{0 . 0 0 0 1}$ \\
AF & 10 & 8 & $\mathrm{p}=0.21$ \\
Diabetes mellitus & 2 & 2 & $\mathrm{NS}$ \\
Heart failure & 0.4 & 0.3 & $\mathrm{NS}$ \\
Valvular heart disease & 0.2 & 0.2 & $\mathrm{NS}$ \\
Anticoagulation & 0.4 & 0.9 & $\mathrm{NS}$ \\
Aspirin & 34 & 35 & $\mathrm{NS}$ \\
ACE I/ARB & 51 & 34 & $\mathbf{p}<\mathbf{0 . 0 0 0 1}$ \\
Beta blockers & 25 & 17 & $\mathbf{p}=\mathbf{0 . 0 0 0 2}$ \\
Statin & 43 & 40 & $\mathrm{p}=0.36$ \\
Ejection fraction & $\mathbf{6 5} \pm \mathbf{5}$ & $\mathbf{p}=\mathbf{0 . 0 0 1}$ \\
Left atrial volume & $\mathbf{3 1} \pm \mathbf{1 0}$ & $\mathbf{6 4} \pm \mathbf{5}$ & $\mathbf{p}=\mathbf{0 . 0 2}$ \\
METs & $10.9 \pm 3.1$ & $\mathbf{3 0} \pm \mathbf{8}$ & $\mathrm{p}=0.55$ \\
\hline
\end{tabular}


BMI - Body mass index, BSA - Body surface area, LVH - left ventricular hypertrophy, AF - Atrial fibrillation, ACE I - Angiotensin converting enzyme inhibitor, ARB - Angiotensin receptor blocker, METs Metabolic equivalents, ECG - electrocardiogram

Figures.

Figure Legends.

Figure 1. Outcomes (combined composite events) for patients with any stress test ST depression compared to those with none.

Figure 2. Outcomes (combined composite events) for patients with exercise ST depression [?] 1.5mm compared to those without ST segment change.

Figure 3. Comparison of prognostic risk based on amount of exercise induced horizontal of downsloping ST depression (unadjusted and adjusted).

Figure 4. Major adverse cardiac outcomes (death, acute coronary syndromes and revascularisation) for with exercise induced ST depression of [?] 1.5mm compared to those without ST segment change.

Figure 5. Outcomes (combined composite events) for patients with exercise induced ST depression of [?] 1.5mm and exercise [?] 13 METs compared to those with exercise to [?] 13 METs.

Figure 1. Outcomes (combined composite events) for patients

with any stress test ST depression compared to those with none

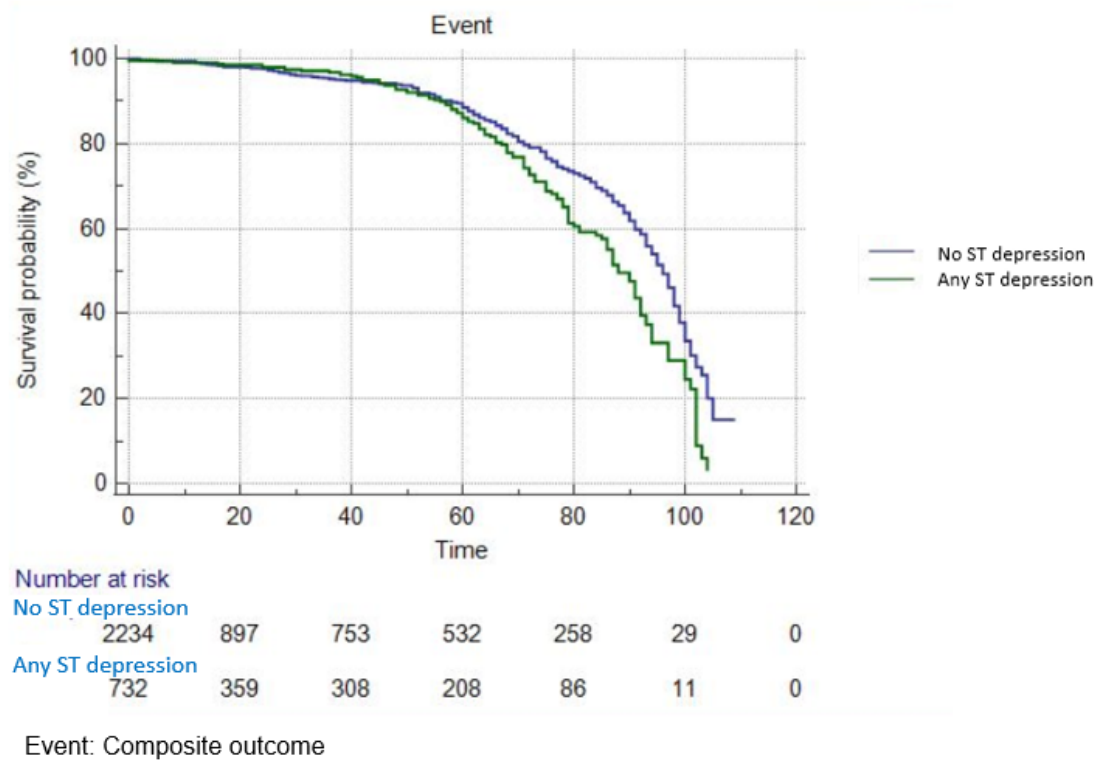


Figure 2. Outcomes (combined composite events) for patients

with exercise induced ST depression of $\geq 1.5 \mathrm{~mm}$ compared to

those without ST segment change

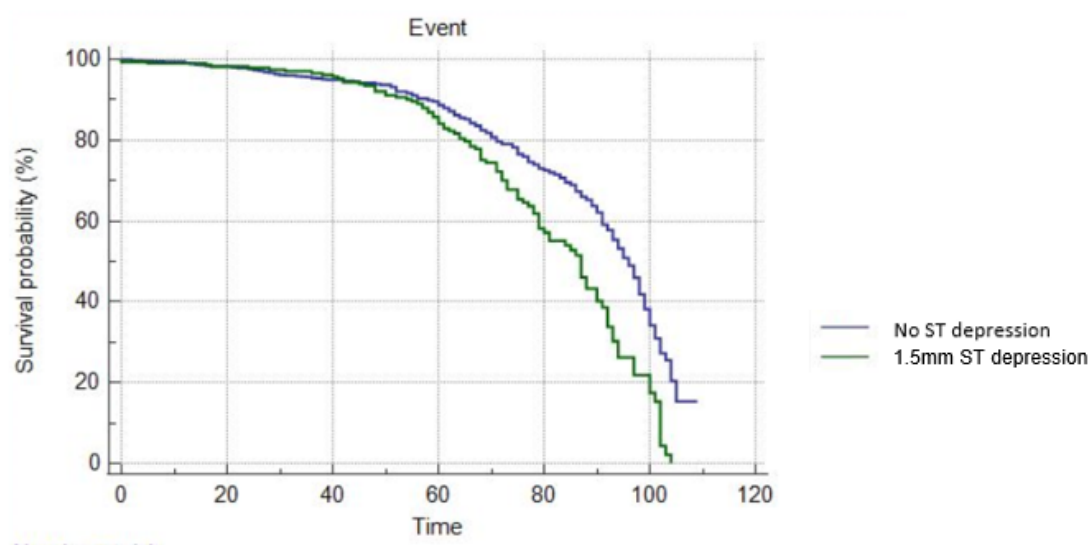

Number at risk

No ST depression

$\begin{array}{llllll}2470 \quad 1003 & 848 & 598 & 288 & 32 & 0 \\ \begin{array}{l}\text { ST depression } \\ 496\end{array} \quad 253 & 213 & 142 & 56 & 8 & 0\end{array}$

Event: Composite outcome

\begin{tabular}{|c|c|c|c|c|c|c|}
\hline \multirow{2}{*}{\multicolumn{7}{|c|}{$\begin{array}{l}\text { Unadjusted for } \\
\text { risk }\end{array}$}} \\
\hline & & & & & & \\
\hline $1 \mathrm{~mm}$ & 732 & 1.55 & $1.22-1.95$ & $\mathrm{P}<0.0 .0001$ & & \\
\hline $1.5 \mathrm{~mm}$ & 497 & 1.73 & $1.37-2.20$ & $p<0.0001$ & & \\
\hline $2 \mathrm{~mm}$ & 312 & 1.63 & $1.22-2.18$ & $p=0.0009$ & & \\
\hline $3 \mathrm{~mm}$ & 78 & 1.94 & $1.16-3.21$ & $p=0.011$ & & \\
\hline \multicolumn{7}{|l|}{ Adjusted for risk } \\
\hline $1 \mathrm{~mm}$ & 732 & 0.48 & $0.11-0.82$ & $p=0.94$ & & 1 \\
\hline $1.5 \mathrm{~mm}$ & 497 & 1.28 & $1.10 \cdot 2.12$ & $p=0.0001$ & $1 \mathrm{~mm} \quad 1.5 \mathrm{~mm} \quad 2 \mathrm{~mm} \quad 3 \mathrm{~mm}$ & $1 \mathrm{~mm} \quad 1.5 \mathrm{~mm} \rightarrow-2 \mathrm{~mm}$ \\
\hline $2 \mathrm{~mm}$ & 312 & 1.39 & 1.13-1.94 & $p=0.001$ & \multirow{2}{*}{ Hazard ratio uncorrected } & Hazard ratio corrected \\
\hline $3 \mathrm{~mm}$ & 78 & & & NS & & 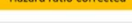 \\
\hline
\end{tabular}

NS - Not significant 
Figure 4. Major adverse cardiac outcomes (death, acute coronary

syndromes and revascularisation) for with exercise induced ST depression

of $\geq 1.5 \mathrm{~mm}$ compared to those without ST segment change

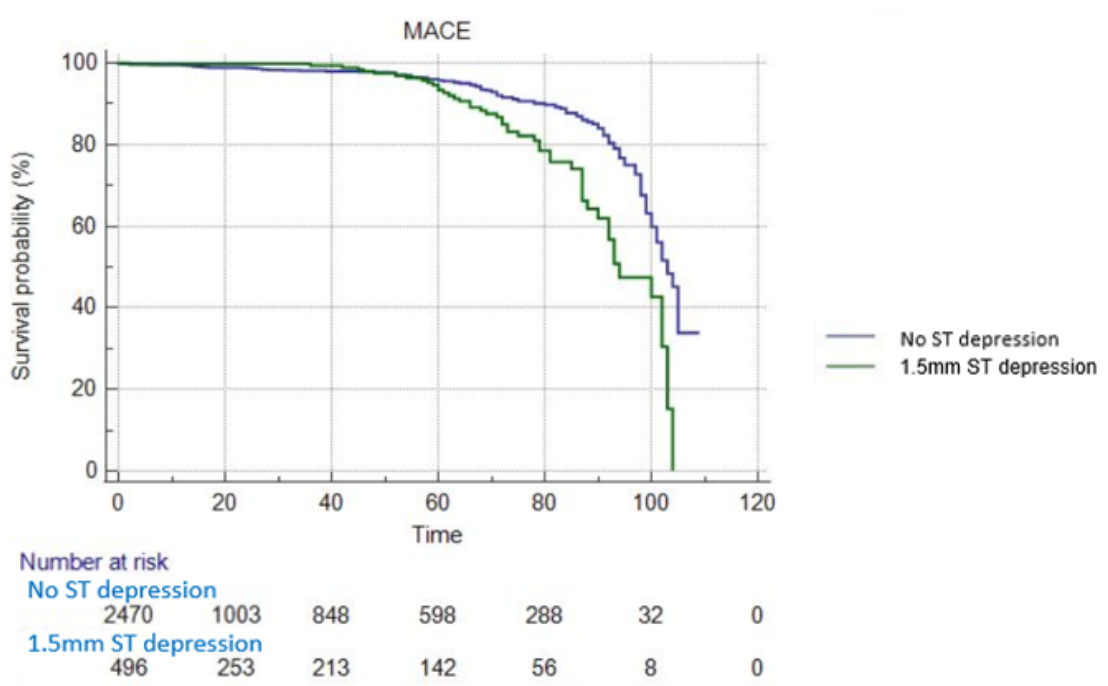

MACE - Major adverse cardiac outcomes

Figure 5. Outcomes (combined composite events) for patients with exercise induced ST depression of $\geq 1.5 \mathrm{~mm}$ and exercise $\geq$ 13 METs compared to those with exercise to $\leq 13 \mathrm{METs}$

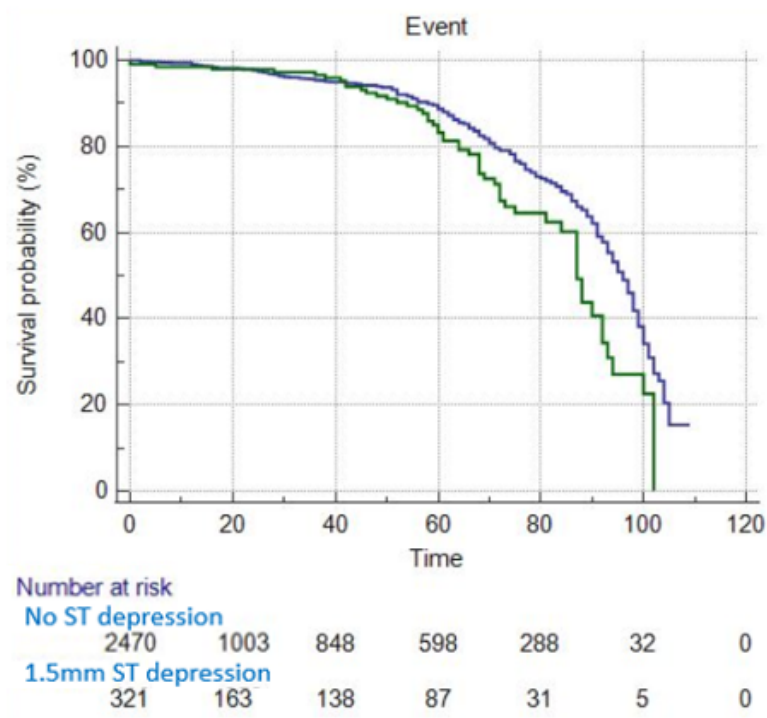

Event: Composite outcome 